\title{
Intraperitoneal Chemotherapy as a Multimodal Treatment for Gastric Cancer Patients with Peritoneal Metastasis
}

\author{
Sachio Fushida*, Katsunobu Oyama, Jun Kinoshita, Tomoya Tsukada, Kouichi Okamoto, \\ Hidehiro Tajima, Itasu Ninomiya, Hirohisa Kitagawa, Takashi Fujimura, Tetsuo Ohta \\ Department of Gastroenterological Surgery, Kanazawa University Hospital, Kanazawa, Japan. \\ Email: *fushida@staff, kanazawa-u.ac.jp
}

Received June $30^{\text {th }}, 2013$; revised July $29^{\text {th }}, 2013$; accepted August $6^{\text {th }}, 2013$

Copyright (C) 2013 Sachio Fushida et al. This is an open access article distributed under the Creative Commons Attribution License, which permits unrestricted use, distribution, and reproduction in any medium, provided the original work is properly cited.

\begin{abstract}
Peritoneal metastasis of gastric cancer is mainly caused by the dispersion of free cancer cells from the serosal surface of the invaded stomach, from surgically transected lymphatic channels, and from tumor cell-containing blood from the primary lesion into the peritoneal cavity. Intraperitoneal chemotherapy (IPC) combined with surgery has performed for the prevention and treatment of peritoneal metastasis in gastric cancer. The efficacy of this technique is influenced by the pharmacokinetic advantage achievable with the anticancer drug, timing of administration, combination with hyperthermia, and tumor volume. The pharmacokinetic advantage for peritoneal cavity exposure relative to peripheral circulation by intraperitoneal delivery for drugs including cisplatin (10-fold advantage), mitomycin C (20- to 30-fold advantage), docetaxel (500-fold advantage), and paclitaxel (1000-fold advantage) has been confirmed. To avoid uneven drug distribution in the peritoneal cavity and the re-growth of residual tumor, it seems to be reasonable to perform IPC perioperatively; however, early perioperative intraperitoneal chemotherapy (EPIC) has a relatively high morbidity rate compared with intraoperative IPC. Hyperthermia has both cytotoxicity of itself and a synergistic effect with anticancer drugs, especially mitomycin C. In the adjuvant setting, patients with either hyperthermic intraperitoneal chemotherapy (HIPEC) or EPIC showed a significant improvement of survival compared to those with surgery alone. In addition, extensive intraoperative peritoneal lavage (EIPL) seems also to be a reasonable method to reduce free cancer cells in the peritoneal cavity. For the treatment of peritoneal metastasis, cytoreductive surgery which achieves R0 or R1 resection followed by IPC has demonstrated a survival benefit, whereas gross residual tumor (R2) treated by IPC has shown poor prognosis. Extensive cytoreductive surgery, such as peritonectomy, followed by IPC achieved long-term survival for selected patients, though this aggressive procedure led to high morbidity and mortality rates. It seems that combined chemotherapy (systemically and intraperitoneally) followed by conversion surgery can be expected to be a powerful procedure for the patients with gross peritoneal tumors.
\end{abstract}

Keywords: Peritoneal Metastasis; Gastric Cancer; Intraperitoneal Chemotherapy

\section{Introduction}

One of the most characteristic features of gastric cancer and the most frequent causes of death from this disease is peritoneal metastasis. In a recent multicentric prospective study [1], the median survival time was 3.1 months for gastric cancer patients. For two decades now, the treatment of peritoneal metastasis has consisted of systemic chemotherapy with sequential methotrexate (MTX) and 5-FU, or IPC with mitomycin C (MMC), cisplatin, OK432, and other agents. Sequential MTX and 5-FU have been widely used as systemic chemotherapy because of

*Corresponding author. their high efficacy against poorly differentiated adenocarcinoma, persistent high concentrations in ascites, and tendency to have low-grade toxicity $[2,3]$. However, gastric cancer is only moderately sensitive to chemotherapy, and peritoneal metastasis is known to be relatively resistant to systemic chemotherapy due to the poor blood supply and oxygenation of cancer cells in the peritoneum. Therefore, in those days to enhance the efficacy of anticancer drugs, IPC has been generally accepted as regional intensive chemotherapy for the prevention and treatment of peritoneal metastasis.

IPC with MMC or carbon-adsorbed MMC has been reported to improve the survival of gastric cancer pa- 
tients by preventing peritoneal recurrence [4], but the efficacy of these therapies in patients with peritoneal metastasis has not been established.

Intraperitoneal (IP) cisplatin treatment has been performed safely and effectively in ovarian cancer by many investigators $[5,6]$.

The usefulness of IP cisplatin must be confirmed by controlled clinical studies, but a multicenter randomized trial, JCOG 9206-2 (Japan Clinical Oncology Group), which was comparing adjuvant IP cisplatin with no IP treatment, could not reveal the superiority of IP cisplatin [7].

Moreover, although even three large randomized phase III trials comparing IP versus intravenous (IV) cisplatin-based chemotherapy have shown a survival benefit of IPC, this approach has not been accepted as a standard treatment for gynecologic tumors [8-10].

HIPEC has been developed since 1980 [11] and MMC is the most frequently used chemotherapeutic agent in anticipation of its synergy effect with hyperthermia [12]. The efficacy of IPC seems to be affected mainly by the extent of the peritoneal tumor and ascites. Most of positive results were obtained for patients without peritoneal metastasis in an adjuvant setting or for patients with microscopic residual tumor [13-22].

Until recently, systemic chemotherapy was regarded as less effective than IPC against peritoneal metastasis; however, novel drugs, such as S-1 and taxanes, are expected to produce a good outcome despite the existence of the blood-peritoneal barrier.

S-1 is a novel oral dehydropyrimidine derivative of 5$\mathrm{FU}[23,24]$. The response rate for S-1 in gastric cancer is over $40 \%$, and S-1 has the potential to prolong survival in advanced gastric cancer $[25,26]$. Moreover, S-1, unlike other fluoropyrimidine agents, is effective even against peritoneal metastasis. This was confirmed using a mouse model developing peritoneal metastasis of gastric cancer [27]. Although the detailed mechanism is unknown, S-1 appears to supply the peritoneal tumor with 5-fluorouracil via the systemic and intraperitoneal circulation. Considering the efficacy and survival benefit of S-1 in patients with peritoneal metastasis, which have been documented by many case reports and other reports [28, 29], it will be necessary to include S-1 in the regimen of a randomized study for treatment of peritoneal metastasis.

Taxanes such as docetaxel and paclitaxel bind to tubulin, leading to microtubule stabilization, mitotic arrest and, subsequently, cell death $[30,31]$. The activity of taxanes may depend on the property of killing tumor cells in the absence of wild-type p53 function [32], unlike other drugs requiring wild-type $\mathrm{p} 53$, and taxanes may therefore be effective against gastric cancer cells, which frequently have p53 mutations [33,34]. Furthermore, these compounds have high sensitivity against poorly differentiated adenocarcinoma, which is a common type of peritoneal tumor, and some of these compounds, when administered intravenously, are transported into the peritoneal cavity $[35,36]$. These findings suggest that taxanes are also candidates for first-line drugs for peritoneal metastasis.

Because of the large body of data showing the efficacy for peritoneal metastasis of either S-1 or taxanes administered as systemic chemotherapy, oncologists may not be very likely to consider IPC.

This article will briefly review the current status of IPC, with a particular focus on pharmacokinetics, treatment timing, and tumor volume and combination therapy. It is hoped that this review will help renew the interest of oncologists in IPC.

\section{Rationale for Intraperitoneal Chemotherapy}

Administration of anticancer drug into the peritoneal cavity is one of the types of regional therapy, and its merit is that it exposes peritoneal lesions to high concentrations of drugs for more prolonged periods than systemic treatment [37]. The agents for intraperitoneal administration are required to be effective against the target tumor and to show enhanced cytotoxicity with either increased drug concentration or exposure duration. If an individual tumor has not responded to systemic therapy, even 10-fold or higher increases in drug concentration are not expected to achieve a good outcome via regional therapy [38].

\section{Pharmacokinetics and Local Toxicity}

The pharmacokinetic properties of many anticancer agents have been examined, following intraperitoneal administration in phase I studies [38] (Table 1). Ideal agents for intraperitoneal delivery have a high ratio of either peak peritoneal drug concentration or area under the peritoneal concentration versus time curve (AUC) relative to systemic concentration, i.e., they have a low peritoneal clearance and a high plasma clearance. Such a pharmacokinetic advantage for peritoneal cavity exposure is favored by high molecular weight, water solubility, high solution volume, and easy ionization. The pharmacokinetic advantage has been reported to range from 10-fold for cisplatin [39] and carboplatin [40], to as high as 1000 -fold for paclitaxel [41].

Because MMC, which is commonly used intraperitoneally, is rapidly absorbed through capillary walls in the subperitoneum due to its low solubility in water, MMC has only a 20 - 30-fold pharmacokinetic advantage and disappears from the blood within 3 hours [42]. 
Table 1. Area under the curve ratios of intraperitoneal exposure to systemic agent [38].

\begin{tabular}{cc}
\hline Drugs & Area under the curve ratio \\
\hline 5-Fluorouracil & 250 \\
Carboplatin & 10 \\
Cisplatin & 7.8 \\
Docetaxel & 550 \\
Doxorubicin & 230 \\
Etoposide & 65 \\
Gemcitabin & 500 \\
Mitomycin C & 23.5 \\
Oxaliplatin & 16 \\
Paclitaxel & 1000 \\
\hline
\end{tabular}

Cisplatin, whose molecular weight is relatively low, is also rapidly absorbed from subperitoneal capillaries and transported into the systemic circulation. Pharmacokinetic studies confirmed that the plasma AUC is similar after IP or IV cisplatin. Furthermore, adverse effects after IP cisplatin and are also similar to those after IV cisplatin, and include effects such as emesis, nephrotoxicity, and neurotoxicity, and there are minimal local adverse events. Considered together, these findings indicate that IP cisplatin may exert anticancer effects both regionally and systemically [38]. However, it has been shown that cisplatin seems to be useful for patients with microscopic residual tumor but not for the patients with macroscopic residual tumor. Therefore, it is necessary to search for some other highly effective agent for IP in gastric cancer.

Paclitaxel is retained in the peritoneal cavity at cytotoxic concentrations for at least 7 days, implying that very limited amounts of paclitaxel enter the systemic compartment after IP [43]. This is reasonable considering the fact that the dose-limiting factor for paclitaxel is abdominal pain from direct peritoneal irritation. These findings suggest that the cytotoxic activity of IP paclitaxel may be exerted by direct penetration into the regional tumor alone.

In contrast, docetaxel has a pharmacokinetic advantage of two logs associated with its intraperitoneal delivery, and the systemic AUC after intraperitoneal administration is 2 times greater than that after standard intravenous administration $[44,45]$. These data indicate that docetaxel occupies a position between cisplatin and paclitaxel from the pharmacokinetic viewpoint (Figure 1). Paclitaxel and docetaxel have rather similar chemical and physiological characteristics, and the pharmacokinetic difference between them seems to be attributable to the differential absorption in solubility [44]. The injection preparation of paclitaxel, Taxol, contains a high concentration of Cremophor EL as the surfactant vehicle, which suppresses

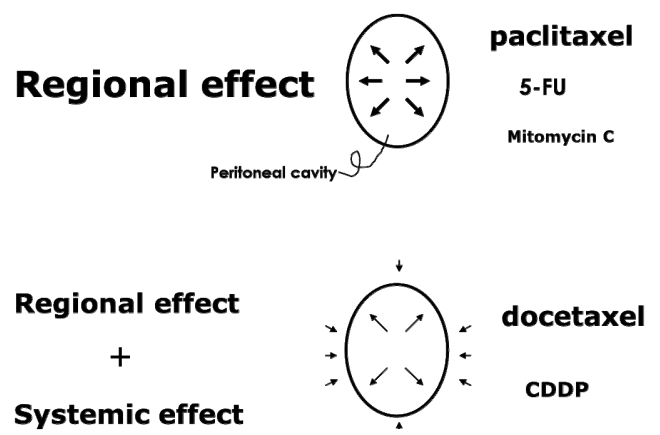

Figure 1. Anticancer effect according to pharmacokinetic difference.

the permeation of the anticancer drug into tissues and cells, and this may explain why intraperitoneally administered Taxol shows lower systemic transportation than that of Taxotere, which contains a low concentration of Polysorbate- 80 as the surfactant [46].

In the selection of anticancer drugs for IPC, it is necessary to consider to not only pharmacokinetic advantage but also local toxicity due to administrated drug. Drugs which possess serosa-damaging activity should be avoided to use because their drugs, such as MMC and adriamycin, would induce the encapsulated chemical peritonitis, which represent for peritoneal fibrosis and intestinal adhesion, associated with poor delivery (Figure 2).

\section{The Treatment Schedule of Perioperative IPC}

The issue of when IPC should be administered is also important with respect to the treatment efficacy. Sautner et al. [47] reported that adjuvant IP cisplatin between postoperative day 10 and 28 dose not improve long-term survival. This means that the best time to perform chemotherapy is just after cytoreduction surgery because the remaining cancer burden is the smallest at that time. The growth of cancer cells shed into the peritoneal cavity is protected by the forming a connective tissue matrix and is stimulated by cytokines in the healing surgical wound [48]. Moreover, the delaying initiation of IPC after surgery leads to poor delivery of anticancer agents caused by peritoneal adhesion. For these reasons and for convenience, most surgeons perform intraoperatively in Japan. On the other hand, early postoperative intraperitoneal chemotherapy (EPIC), which is performed for $5-7$ days from postoperative day 1 with 5-FU, MMC or cisplatin $[17,49]$, is also performed in western countries and Korea. EPIC had not been performed until Sugarbaker reported this strategy [50], because it was feared that early postoperative chemotherapy might increase the rate of morbidity. Yu et al. [17] reported that there was a significant increase in the incidence of intraabdominal bleeding and intraabdominal sepsis in the EPIC group 


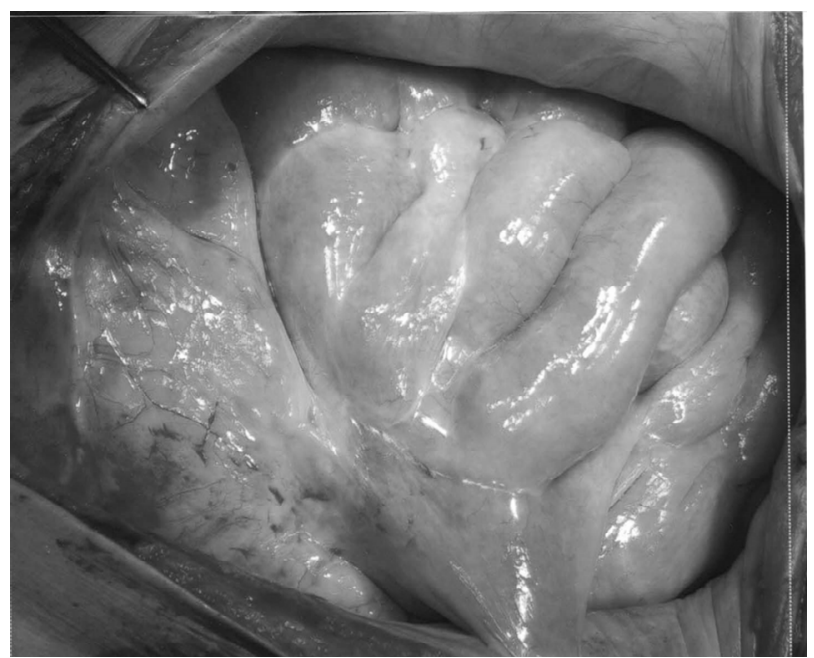

Figure 2. Encapsulated chemical peritonitis after intraperitoneal administration of mitomycin.

compared with the control group, although most of these complications could be managed conservatively.

\section{Infusion Methods}

Intraperitoneally injected drugs are usually distributed unevenly because of the anatomical complexity of the peritoneal cavity. It is considered that about $1000 \mathrm{ml}$ of solution is necessary to adequately distribute the drug into the peritoneal cavity (Figure 3(a)), while excess volume causes a poor outcome due to the low concentration of dissolved agents. For patients with massive ascites, it is better to carry out peritoneal lavage with physiologic saline or potassium hydroxide, which dissolves mucinous retention. Alpha 1-acid glycoprotein in the ascites also reduces the anticancer effect by binding some agents, such as cisplatin and taxanes [51,52].

An intraperitoneal catheter provides an easy method for repeated administration of anticancer drugs, peritoneal lavage, and/or cytological examination (Figure 3(b)), although possible intraperitoneal catheter complications include catheter infection, blocked catheter, and bowel complications. Makhija et al. [53] analyzed complications associated with the use of IP chemotherapy at Memorial Sloan Kettering Cancer Center and reported that out of 411 patients, catheter malfunction occurred in $32(7.8 \%)$ and catheter-induced sepsis occurred in 14 (3.4\%). In gastric cancer, an implanted intraperitoneal access port (Bardport; C.R. Bard, Inc., NJ, USA) has been used generally for IPC. Emoto et al. previously described that although $20.6 \%$ of 131 gastric cancer patients with peritoneal metastases experienced port complications, complications were controllable and chemotherapy was not terminated by complications [54]. Thus, IPC using a port might be safe and feasible under appro-

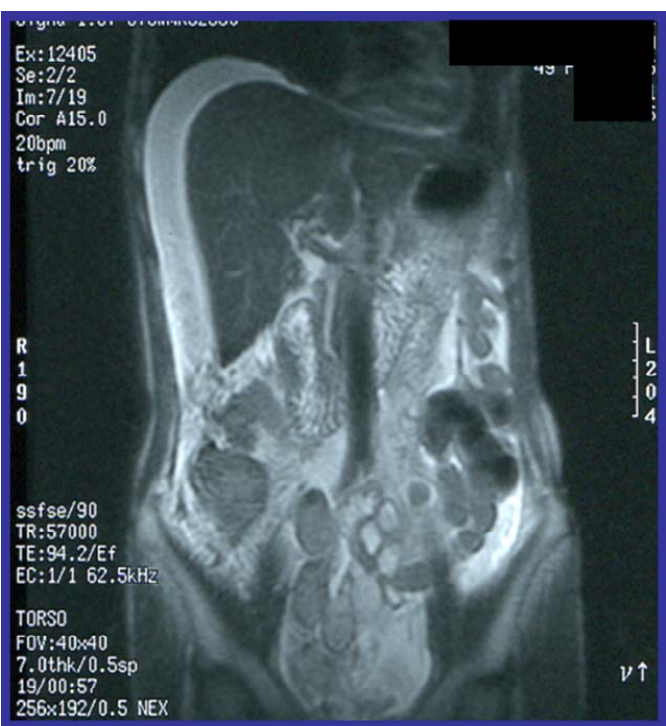

(a)

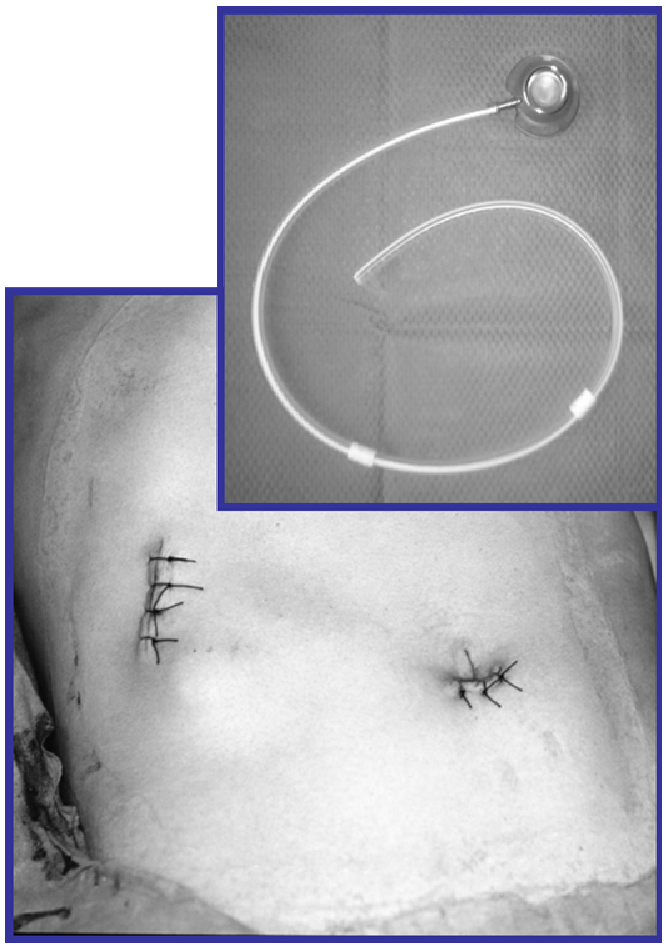

(b)

Figure 3. (a) MRI showed equal distribution of fluid with $1 \mathrm{~L}$ of saline containing anticancer agent; (b) Intraperitoneal access port and its implanted view subcutaneously.

priate management.

\section{Combination with Hyperthermia}

In the 1980s, HIPEC was reported [55] as a safe treatment for peritoneal metastasis, and it is currently under evaluation. Hyperthermia has been developed as an anticancer therapy and has been employed clinically for its 
direct cytotoxic effect [56] and synergy with some types of chemotherapeutic agents. The detailed mechanisms by which hyperthermia enhances the cytotoxicity of MMC are unclear but include increased cellular accumulation of MMC, increased activation of MMC, and altered repair of DNA damage caused by MMC [57].

It is important to note that HIPEC is not a very powerful treatment for patient with macroscopic residual tumors, and that it has the disadvantage of requiring complicated procedures to maintain the appropriate temperature. Therefore, cytoreductive surgery followed by HIPEC might be recommended in that situation. A recent phase III study showed that patients treated with CRS plus HIPEC had superior survival than those treated with CRS alone, and the median survival time (MST) for CRS plus HIPEC was 11.0 months [58].

\section{Prophylactic (Adjuvant) Chemotherapy}

In an adjuvant setting, IPC is designed to eradicate residual microscopic tumor and floating cancer cells in the peritoneal cavity after curative resection.

Some prospective randomized studies of IPC in the adjuvant setting have yielded various results (Table 2). IPC with MMC or carbon-adsorbed MMC has been reported to improve survival of gastric cancer patients by preventing peritoneal recurrence $[4,5]$, but the efficacy of these therapies was not confirmed by the Austrian Gastric Cancer Working Group [59]. Fujimura et al. [14] have showed that patients treated with intraoperative HIPEC with cisplatin, MMC and etoposide had longer survival than the control group, but this result was not confirmed by Kunisaki et al. [60]. These discrepant data seem to be influenced by the number of free cancer cells disseminated during surgical treatment.

Dissemination of cancer cells by surgical manipulation of tumors with serosal invasion and leakage of lymph containing cancer cells from the transected lymphatic channels may be unavoidable during gastrectomy. Although free cancer cells in the peritoneal cavity play an important role in peritoneal recurrence, the sensitivity of detecting them is often influenced by the methodology, such as morphological cytology, immunological cytostaining or RT-PCR [61]. Therefore, adjuvant IPC should be performed for patients with serosal invasion. However, it is difficult to detect the metastatic lymph nodes as another source of free cancer cells intraoperatively. Investigators [17] who recommended EPIC advocate that because the pathologic stage can be determined only postoperatively, selective adjuvant IPC should be done postoperatively.

For the prevention of peritoneal recurrence, Shimada and colleague $[62,63]$ reported that extensive intraoperative peritoneal lavage (EIPL) followed by IPC is useful for eradicating free cancer cells in the peritoneal cavity and micrometastases on the peritoneal surface. Because free cancer cells may be reduced to almost zero using this method, treatment evaluation would not be influenced by differences of the number of free cancer cells between patients. Although JCOG9206-2 study showed no significant difference of survival rate between IP cisplatin group and non-IP group [7], it was reason why that peritoneal lavage before administration of cisplatin might be insufficiently. On the other hand, in patients with positive peritoneal cytology and no macroscopic peritoneal tumor, radical surgery followed by postoperative S-1 showed good results with 2-year survival rate of $46 \%$ and 5 -year survival rate 26\% [64]. Further investigation, including controlled clinical trials comparing S-1 and EIPL plus IP cisplatin followed by S-1, are needed.

\section{Intraoperative Chemotherapy for Peritoneal Carcinomatosis}

Reducing tumor volume has always been considered an important factor in achieving tumor response to chemotherapy. Glehen et al. [20] reported that with combined HIPEC, patients treated with complete or sub-complete surgery had significantly longer survival than those with incomplete cytoreduction. Similar results were reported by Yonemura et al. [19], who showed that postoperative survival after cytoreductive surgery was inversely related with the residual tumor burden. He also described that according to peritoneal cancer index (PCI), which established by Sugarbaker as a semiquantitative scoring system [65] (Figure 4), it was difficult to complete cytoreduction in the patients with PCI $\leq 6$ [66]. When cytoreductive surgery does not result in sufficient down-staging, the survival benefit of HIPEC remains extremely low, and median survival does not exceed 6 to 8 months.

On the basis of these data, peritonectomy was first described as a new cytoreductive surgery technique for gross tumors in 1995 [67]. This procedure consists of five steps: epigastric peritonectomy, anterolateral peritonectomy, subphrenic peritonectomy, omental bursa peritonectomy, and pelvic peritonectomy. These extensive treatments have been developed in order to remove all macroscopic lesions to enhance the efficacy of HIPEC. This aggressive treatment achieved the long term survival for selected diseases, such as pseudomyxoma peritonei and some colorectal cancers $[68,69]$. However, the combination of peritonectomy and HIPEC can lead to greater mortality and morbidity rates. Improvement of the morbidity rate of the peritonectomy procedure requires more experience with this operation in not only the surgical technique of peritonectomy but also the postoperative intensive care of the patients [70]. At the moment, extensive cytoreductive surgery of peritoneal gross tumors is not the standard procedure in the management of gastric cancer. To reduce the high rate of morbidity 
Table 2. Intraperitoneal chemotherapy after curative resection for gastric cancer.

\begin{tabular}{cccc}
\hline Author & regimen & hyper-thermia & $\begin{array}{c}\text { survival rate } \% \text { (year) } \\
\text { study/control }\end{array}$ \\
\hline R. Hamazoe [13] & MMC & yes & $61 / 53(5)$ \\
T. Fujimura [14] & MMC/CDDP/Etoposide & yes & $68 / 23(3)$ \\
T. Takahashi [15] & MMC-Carbon & no & $38 / 20(3)$ \\
S. Fujimoto [16] & MMC & yes & $69 / 55(5)$ \\
W. Yu [17] & MMC/5FU & no & $54 / 38(5)$ \\
Y. Yonemura [18] & MMC/CDDP/Etoposide & yes & $61 / 42(5)$ \\
T. Sautoner [47] & CDDP & no & yes \\
H. R. Rosen [59] & MMC-Carbon & no & yes \\
C. Kunisaki [60] & MMC/CDDP/Etoposide & yes & yes \\
\hline
\end{tabular}

Abbreviation: MST, median survival time; N/A, not available.

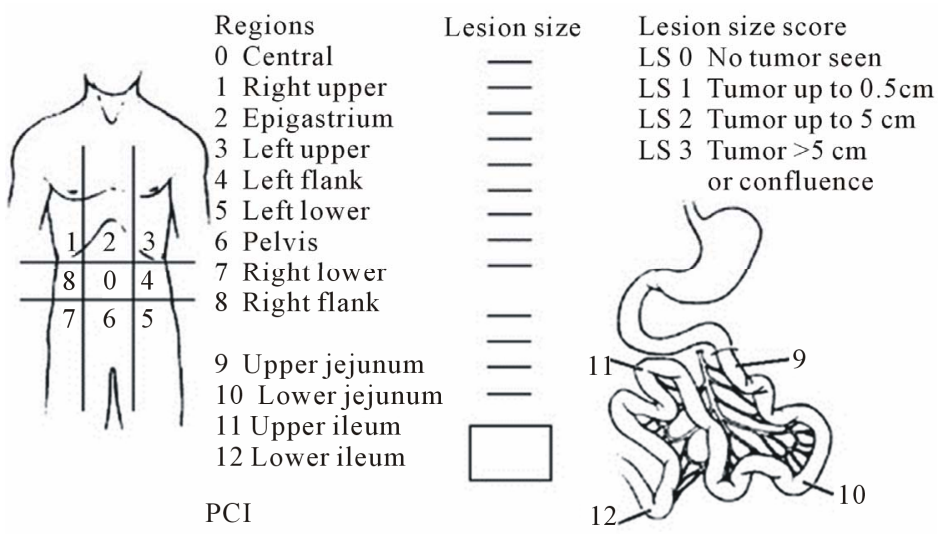

Figure 4. Peritoneal cancer index (PCI). Peritoneal cavity is divided into 13 parts, which ranges from 0 to 12 . Accurate measurement of each region is scored as lesion size 0 through 3. LS 0: no implant.

from extensive cytoreduction, an appropriate neoadjuvant approach is needed prior to surgery.

\section{Neoadjuvant Chemotherapy}

For accurate judgment regarding whether peritoneal metastasis could be expected, laparoscopy may play an important role by allowing direct observation of the peritoneal cavity in spite of the development of imaging techniques such as CT, MRI and PET. Although peritoneal lavage cytology is also useful for the diagnosis of peritoneal metastasis, this cytology cannot provide information about the degree, extent, and volume of peritoneal metastasis. If the laparoscopic examination reveals unresectable peritoneal metastasis, induction chemotherapy would be recommended. Considering that the actual depth of penetration of drugs injected intraperitoneally directly into the tumor is limited to $2-3 \mathrm{~mm}$ from the peritoneal surface [71], it seems to be necessary to attack both systemically and regionally.

For the following salvage surgery, intraperitoneal drug should have sufficient efficacy against the tumor and should not induce peritonitis or adhesion despite repeated administration as well as taxanes. Taxanes have high sensitivity against diffuse-type adenocarcinoma, which is a common type of peritoneal tumor. Furthermore, taxanes are absorbed through the openings of lymphatic system, such as the milky spots and the stomata which are important sites for the formation of peritoneal metastases [72], due to their large molecular weight and fat solubility. The oral anticancer drug S-1 is a fluoropyrimidine derivative, combining tegafur with two modulators. S-1 is also highly effective against gastric peritoneal metastasis due to the higher concentration of 5-FU and CDHP achieved in peritoneal tumors than in plasma. These findings suggest that combination therapy of S-1 plus IP taxanes should be the first-line for PC.

According this sense, Ishigami et al. established IP paclitaxel with S-1 plus IV paclitaxel and provided encourage results with a MST of 22.5 months and 2-year survival rate of $78 \%$ [73]. Peritoneal metastasis is considered to be a non-measurable lesion because it is difficult to detect peritoneal metastasis by conventional radiological examinations. New response criteria for treatment against peritoneal metastasis were developed according to the findings of intraperitoneal photographs which were taken in the first and second laparoscopy [45]. In the phase II study to evaluate efficacy of S-1 plus IP docetaxel [74], the second staging laparoscopy after 2 cycles of combined chemotherapy showed response rate of $52 \%$ according to the response criteria for the treat- 
ment of peritoneal metastasis. This study also showed a 1 -year survival rate of $70.4 \%$ with MST of 16.2 months. Peritoneal responder who underwent gastrectomy showed 2-year survival rate of $48 \%$, and non-responder who received chemotherapy alone showed $0 \%$. These data suggest that IP taxane combined with oral administration of S-1 following surgery may be a powerful candidate for the treatment of severe peritoneal metastasis. However, it is important to note that in the absence of data from randomized trials, the efficacy of this specific multi-modality strategy remains to be established.

\section{Conclusion}

The development of multimodal treatment consisting of neo-adjuvant chemotherapy, cytoreductive surgery, perioperative IPC, and adjuvant chemotherapy is expected because chemotherapy alone is not sufficient for complete remission of peritoneal metastasis. Although Yonemura et al. developed the most aggressive multimodal treatment combining neoadjuvant intraperitoneal-systemic chemotherapy (NIPS), peritonectomy, HIPEC, and EPIC [66], it is difficult to start phase III clinical trials using this treatment because of the high morbidity and mortality rates. In the meantime, Ishigami et al. conducted a randomized phase III trial, so called PHOENIXGC trial, comparing combination of S-1, IV paclitaxel and IP paclitaxel with S-1/cisplatin, which is the current standard regimen for gastric cancer patients with peritoneal metastasis in Japan. Most of the patients who received this intraperitoneal and systemic chemotherapy were underwent R0 resection and prolonged survival with low morbidity and mortality in Phase II trial. Therefore, the forthcoming results of PHOENIX-GC trial are expected to revolutionize treatment of peritoneal metastasis in gastric cancer.

\section{REFERENCES}

[1] B. Sadeghi, C. Arvieux, O. Glehen, et al., "Peritoneal Carcinomatosis from Non-Gynecologic Malignancies: Results of the EVOCAPE 1 Multicentric Prospective Study," Cancer, Vol. 88, No. 15, 2000, pp. 360-365.

[2] T. Konishi, T. Noie, J. Yoshida, et al., "Treatment of Peritoneal Dissemination of Gastric Cancer with Sequential Methotrexate and 5-Fluorouracil," Gastric Cancer, Vol. 2, No. 1, 1999, pp. 52-56. http://dx.doi.org/10.1007/s101200050021

[3] M. Tahara, A. Ohtsu, N. Boku, et al., "Sequential Methotrexate and 5-Fluorouracil Therapy for Gastric Cancer Patients with Peritoneal Dissemination: A Retrospective Study," Gastric Cancer, Vol. 4, No. 4, 2001, pp. 212-218. http://dx.doi.org/10.1007/s10120-001-8012-x

[4] A. Hagiwara, T. Takahashi, O. Kojima, et al., "Prophylaxis with Carbon-Adsorbed Mitomycin against Peritoneal Recurrence of Gastric Cancer," Lancet, Vol. 339, No.
8794, 1992, pp. 629-631. http://dx.doi.org/10.1016/0140-6736(92)90792-2

[5] S. B. Howell, "Intraperitoneal Chemotherapy for Ovarian Carcinoma," Journal of Clinical Oncology, Vol. 6, No. 11, 1988, pp. 1673-1675.

[6] E. S. Casper, D. P. Kslsen, N. W. Alcock, et al., "Ip Cisplatin in Patients with Malignant Ascites: Pharmacokinetic Evaluation and Comparison with the IV Route," Cancer Treatment Reports, Vol. 67, No. 3, 1983, pp. 235238.

[7] I. Miyashiro, H. Furukawa, M. Sasako, et al., "Randomized Clinical Trial of Adjuvant Chemotherapy with Intraperitoneal and Intravenous Cisplatin Followed by Oral Fluorouracil (UFT) in Serosa-Positive Gastric Cancer versus Curative Resection Alone: Final Results of the Japan Clinical Oncology Group Trial JCOG9206-2," Gastric Cancer, Vol. 14, No. 3, 2011, pp. 212-218. http://dx.doi.org/10.1007/s10120-011-0027-3

[8] D. S. Alberts, P. Y. Liu, E.V. Hannigan, et al., "Intraperitoneal Cisplatin Plus Intravenous Cyclophosphamide versus Intravenous Cisplatin Plus Intravenous Cyclophosphamide for Stage III Ovarian Cancer," New England Journal of Medicine, Vol. 335, No. 26, 1996, pp. 1950 1955. http://dx.doi.org/10.1056/NEJM199612263352603

[9] M. Markman, B. N. Bundy, D. S. Albert, et al., "Phase III Trial of Standard-Dose Intravenous Cisplatin Plus Paclitaxel versus Moderately High-Dose Carboplatin Followed by Intravenous Paclitaxel and Intraperitoneal Cisplatin in Small-Volume Stage III Ovarian Carcinoma: An Intergroup Study of the Gynecologic Oncology Group, Southwestern Oncology Group, and Eastern Cooperative Oncology Group," Journal of Clinical Oncology, Vol. 19, No. 4, 2001, pp. 1001-1007.

[10] D. K. Armstrong, B. N. Bundy, L. Wenzel, et al., "Intraperitoneal Cisplatin and Paclitaxel in Ovarian Cancer," New England Journal of Medicine, Vol. 354, No. 1, 2006, pp. 34-43. http://dx.doi.org/10.1056/NEJMoa052985

[11] J. S. Spratt, R. A. Adock, M. Muskovin, et al., "Clinical Delivery System for Intaperitoneal Hyperthermic Chemotherapy," Cancer Research, Vol. 40, No. 2, 1980, pp. 256-260.

[12] B. A. Teicher, C. D. Kowai, K.A. Kennedy, et al., "Enhancement by Hyperthermia of the in Vitro Cytotoxicity of Mitomycin C toward Hypoxic Tumor Cells," Cancer Research, Vol. 41, No. 3, 1981, pp. 1096-1099.

[13] R, Hamazoe, M. Maeta and N. Kaibara, "Intraperitoneal Thermochemotherapy for Prevention of Peritoneal Recurrence of Gastric Cancer. Final Results of a Randomized Controlled Study," Cancer, Vol.73, No. 8, 1994, pp. 2048-2052. http://dx.doi.org/10.1002/1097-0142(19940415)73:8<204 8::AID-CNCR2820730806>3.0.CO;2-Q

[14] T. Fujimura, Y. Yonemura, K. Muraoka, et al., "Continuous Hyperthermic Peritoneal Perfusion for the Prevention of Peritoneal Recurrence of Gastric Cancer," World Journal of Surgery, Vol. 18, No. 1, 1994, pp. 150-155. http://dx.doi.org/10.1007/BF00348209

[15] T. Takahashi, A. Hagiwara, M. Shimotsuma, et al., "Prophylaxis and Treatment of Peritoneal Carcinomatosis: In- 
traperitoneal Chemotherapy with Mitomycin C Bound to Activated Carbon Particles," World Journal of Surgery, Vol. 19, No. 4, 1995, pp. 565-569.

http://dx.doi.org/10.1007/BF00294724

[16] S. Fujimoto, M. Takahashi, T. Mutou, et al., "Successful Intraperitoneal Hyperthermic Chemoperfusion for the Prevention of Postoperative Peritoneal Recurrence in Patith Advanced Gastric Carcinoma," Cancer, Vol. 85, No. 3, 1999, pp. 529-534. http://dx.doi.org/10.1002/(SICI)1097-0142(19990201)85: 3<529::AID-CNCR3>3.0.CO;2-9

[17] W. Yu, I. Whang, H. Y. Chung, et al., "Indications for Early Postoperative Intraperitoneal Chemotherapy of Advanced Gastric Cancer: Results of a Prospective Randomized Trial," World Journal of Surgery, Vol. 25, No. 8, 2001, pp. 985-990.

http://dx.doi.org/10.1007/s00268-001-0067-7

[18] Y. Yonemura, X. de Aretxabala, T. Fujimura, et al., "Intraoperative Chemohyperthermic Peritoneal Perfusion as an Adjuvant to Gastric Cancer: Final Results of a Randomized Controlled Study," Hepatogastroenterology, Vol. 48, 2001, pp. 1776-1782.

[19] Y. Yonemura, T. Fujimura, G. Nishimura, et al., "Effects of Intraoperative Chemohyperthermia in Patients with Gastric Cancer with Peritoneal Dissemination," Surgery, Vol. 119, No. 4, 1996, pp. 437-444. http://dx.doi.org/10.1016/S0039-6060(96)80145-0

[20] O. Glehen, V. Schreiber, E. Cotte, et al., "Treatment of Peritoneal Carcinomatosis Arising from Gastric Cancer by Cytoreductive Surgery and Intraperitoneal Chemohyperthermia," Archives of Surgery, Vol. 139, No. 1, 2004, pp. 20-26. http://dx.doi.org/10.1001/archsurg.139.1.20

[21] S. Fujimoto, M. Takahashi, T. Mutou, et al., "Improved Mortality Rate of Gastric Carcinoma Patients with Peritoneal Carcinomatosis Treated with Intraperitoneal Hyperthermic Chemoperfusion Combined with Surgery," Cancer, Vol. 79, No. 5, 1997, pp. 884-891. http://dx.doi.org/10.1002/(SICI)1097-0142(19970301)79: 5<884::AID-CNCR3>3.0.CO;2-C

[22] A. C. Beaujard, O. Glehen, J. L. Caillot, et al., "Intraperitoneal Chemohyperthermia with Mitomycin C for Digestive Tract Cancer Patients with Peritoneal Carcinomatosis," Cancer, Vol. 88, No. 11, 2000, pp. 1375-1382. http://dx.doi.org/10.1002/1097-0142(20000601)88:11<25 12::AID-CNCR12>3.0.CO;2-J

[23] T. Shirasaka, Y. Shimamoto, H. Ohshimo, et al., "Development of a Novel Form of an Oral 5-Fluorouracil Delivative $(\mathrm{S}-1)$ Directed to the Potentiation of the Tumor Selective Cytotoxicity of 5-Fluorouracil by Two Biochemical Modulatiors," Anticancer Drugs, Vol. 7, No. 5, 1996, pp. 548-557.

http://dx.doi.org/10.1097/00001813-199607000-00010

[24] T. Takechi, A. Fujioka, E. Matsushima, et al., "Enhancement of the Antitumor Activity of 5-Fluorouracil (5-Fu) by Inhibiting Dehydropyrimidine Dehydrogenase Activity (DPD) Using 5-Chloro-2,4-Dehydroxypyrimidine (Cdhp) in Human Tumour Cells," European Journal of Cancer, Vol. 38, No. 9, 2002, pp. 1271-1277. http://dx.doi.org/10.1016/S0959-8049(02)00048-5
[25] Y. Sakata, A. Ohtsu, N. Horikoshi, et al., "Late Phase II Study of Novel Oral Fluoropyrimidine Anticancer Drug S-1 (1M Tegafur-0.4M Gimestat-1M Otastat Potassium) in Advanced Gastric Cancer Patients," European Journal of Cancer, Vol. 34, No. 11, 1998, pp. 1715-1720. http://dx.doi.org/10.1016/S0959-8049(98)00211-1

[26] W. Koizumi, M. Kurihara, S. Nakano, et al., "The S-1 Gastrointestinal Cancer Study Group: Phase II Study of S-1, a Novel Derivative 5-Fluorouracil, in Advanced Gastric Cancer," Oncology, Vol. 58, No. 3, 2000, pp. 191197. http://dx.doi.org/10.1159/000012099

[27] T. Mori, Y. Fujiwara, M. Yano, et al., "Experimental Study to Evaluate the Usefulness of S-1 in a Model of Peritoneal Dissemination of Gastric Cancer," Gastric Cancer, Vol. 6, Supplement 1, 2003, pp. 13-18. http://dx.doi.org/10.1007/s10120-003-0226-7

[28] H. Osugi, N. Takeda, M. Takemura, et al., "Oral Fluoropyrimidine Anticancer Drug S-1 for Gastric Cancer Patients with Peritoneal Dissemination," Oncology Reports, Vol. 9, No. 4, 2002, pp. 811-815.

[29] T. Yoshikawa, S. Yanoma, A. Tsuburaya, et al., "Effects of TS-1 on Peritoneal Dissemination of Gastric Cancer in Nude Mice," Hepatogastroenterology, Vol. 51, No. 59, 2004, pp. 1554-1557.

[30] E. K. Rowinsky, L. A. Cazenave, R. C. Donehower, "Taxol: A Novel Investigational Antimicrotubule Agent," Journal of the National Cancer Institute, Vol. 82, No. 15, 1990, pp. 1247-1259. http://dx.doi.org/10.1093/jnci/82.15.1247

[31] I. Ringel and B. Horwits, "Studies with RP56976 (Taxotere): A Semisynthetic Analogue of Taxol," Journal of National Cancer Institute, Vol. 83, No. 4, 1991, pp. 288291. http://dx.doi.org/10.1093/jnci/83.4.288

[32] A. F. Wahl, K. L. Donaldson and C. Fairchild, "Loss of Normal p53 Function Confers Sensitization to Taxol by Increasing G2/M Arrest and Apoptosis," Nature Medicine, Vol. 2, No. 1, 1996, pp. 72-79. http://dx.doi.org/10.1038/nm0196-72

[33] S. Fushida, T. Urano, H. Tsuruta, et al., "Frequent p53 Mutations on Exons 5 and 8 in Human Primary Gastric Cancer," International Journal of Oncology, Vol. 2, No. 4, 1993, pp. 563-568.

[34] E. Tahara, S. Semba and H. Tahara, "Molecular Observations in Gastric Cancer," Seminars in Oncology, Vol. 23, No. 3, 1996, pp. 307-315.

[35] M. Mai, Y. Sakata, R. Kanamaru, et al., "A Late Phase II Clinical Study of RP56976 (Docetaxel) in Patients with Advanced or Recurrent Gastric Cancer: A Cooperative Study Group Trial (Group B)," Japanese Journal of Cancer Chemotherapy, Vol. 26, No. 4, 1999, pp. 487-496.

[36] H. Naitoh, A. Kawaguchi, H. Yamamoto, et al., "Mesurement of Docetaxel Concentration in Blood and Ascites after Drop Infusion into Each Vessel and Intraperitoneal Cavity of Gastric Cancer," Japanese Journal of Cancer Chemotherapy, Vol. 31, No. 12, 2004, pp. 20312034.

[37] R. L. Dedrick, C. E. Myers, P. M. Bungay, et al., "Pharmacokinetic Rationale for Peritoneal Drug Administration 
in the Treatment of Ovarian Cancer," Cancer Treatment Reports, Vol. 62, No. 1, 1978, pp. 1-9.

[38] M. Markman, "Intraperitoneal Drug Delivery of Antineoplastics," Drugs, Vol. 61, No. 8, 2001, pp. 1057-1065. http://dx.doi.org/10.2165/00003495-200161080-00003

[39] E. S. Casper, D. P. Kelsen and N. W. Alcock, "IP Cisplatin in Patients with Malignant Ascites: Pharmacokinetics Evaluation and Comparison with the iv Route," Cancer Treatment Reports, Vol. 67, No. 3, 1983, pp. 325338.

[40] M. W. DeGregorio, B. L. Lum and W. M. Holleran, "Preliminary Observations of Intraperitoneal Carboplatin Pharmacokinetics during a Phase I Study of the Northern California Oncology Group," Cancer Chemotherapy and Pharmacology, Vol. 18, No. 3, 1986, pp. 235-238. http://dx.doi.org/10.1007/BF00273393

[41] M. Markman, E. Rowinsky, T. Hakes, et al., "Phase I Trial of Intraperitoneal Taxol: A Gynecologic Oncology Group Study," Journal of Clinical Oncology, Vol. 10, No. 9, 1992, pp. 1485-1491.

[42] M. Hiratsuka, H. Furukawa, T. Yasuda, et al., "Intraperitoneal Chemotherapy with Mitomycin C or Cisplatin," In: T. Nakajima and T. Yamaguchi, Eds., Multimodality Therapy for Gastric Cancer, Springer, Tokyo, 1999, pp. 58-65. http://dx.doi.org/10.1007/978-4-431-67927-1_8

[43] P. Francis, E. Rowinsky, J. Schneider, et al, "Phase I Feasibility and Pharmacologic Study of Weekly Intraperitoneal Paclitaxel: A Gynecologic Oncology Group Pilot Study," Journal of Clinical Oncology, Vol. 13, No. 12, 1995, pp. 2961-2967.

[44] R. J. Morgan Jr., H. D. James, T. Synold, et al., "Phase I Trial of Intraperitoneal Docetaxel in the Treatment of Advanced Malignancies Primary Confined to the Peritoneal Cavity: Dose-Limiting Toxicity and Pharmacokinetics," Clinical Cancer Research, Vol. 9, No. 1, 2003, pp. 5896-5901.

[45] S. Fushida, J. Kinoshita, Y. Yagi, et al., "Dual AntiCancer Effects of Weekly Intraperitoneal Docetaxel in Treatment of Advanced Gastric Cancer Patients with Peritoneal Carcinomatosis: A Feasible and Pharmacokinetic Study," Oncology Reports, Vol. 19, No. 5, 2008, pp. 1305-1310.

[46] K. Yokogawa, M. Jin, N. Furui, et al., "Disposition Kinetics of Taxanes after Intraperitoneal Administration in Rats and Influence of Surfactant Vehicles," The Journal of Pharmacy and Pharmacology, Vol. 56, No. 5, 2004, pp. 629-634. http://dx.doi.org/10.1211/0022357023303

[47] T. Sautner, F. Hofbauer, D. Depisch, et al., "Adjuvant Intraperitoneal Cisplatin Chemotherapy Does Not Improve Long-Term Survival after Surgery for Advanced Gastric Cancer," Journal of Clinical Oncology, Vol. 12, No. 5, 1994, pp. 970-974.

[48] P. H. Sugarbaker, "Observations Concerning Cancer Spread within the Peritoneal Cavity and Concepts Supporting an Ordered Pathophysiology," Cancer Treatment and Research, Vol. 82, 1996, pp. 79-100.

http://dx.doi.org/10.1007/978-1-4613-1247-5_6

[49] H. C. Jeung, S. Y. Rha, W. I. Jang, et al., "Treatment of
Advanced Gastric Cancer by Palliative Gastrectomy, Cytoreductive Therapy and Postoperative Intraperitoneal Chemotherapy," British Journal of Surgery, Vol. 89, No. 4, 2002, pp. 460-466. http://dx.doi.org/10.1046/j.0007-1323.2001.02048.x

[50] W. J. Cunliffe and P. H. Sugarbaker, "Gastrointestinal Malignancy: Rationale for Adjuvant Therapy Using Early Intraperitoneal Chemotherapy," British Journal of Surgery, Vol. 76, No. 10, 1989, pp. 1082-1090.

http://dx.doi.org/10.1002/bjs.1800761030

[51] R. G. Fish, T. S. Gill, M. Adams, et al., "Serum Haptglobin and Alpha 1-Acid Glycoprotein as Indicators of the Effectiveness of Cis-Diamminedichloroplatinum CDDP) in Ovarian Cancer Patients-A Preliminary Report," European Journal of Cancer \& Clinical Oncology, Vol. 20, No. 5, 1984, pp. 625-630.

[52] R. Bruno, N. Vivler, J. C. Vergniol, et al., "A Population Pharmacokinetic Model for Docetaxel (Taxotere): Model Building and Validation," Journal of Pharmakokinetics \& Biopharmaceutics, Vol. 24, No. 2, 1996, pp. 153-172. http://dx.doi.org/10.1007/BF02353487

[53] S. Makhija, M. Leitao, P. Sabbatini, et al., "Complications Associated with Intraperitoneal Chemotherapy Catheters," Gynecologic Oncology, Vol. 81, No. 1, 2001, pp. 77-81.

[54] S. Emoto, H. Ishigami, A. Hidemura, et al., "Complication and Management of an Implanted Intraperitoneal Access Port System for Intraperitoneal Chemotherapy for Gastric Cancer with Peritoneal Metastasis," Japanese Journal of Clinical Oncology, Vol. 42, No.11, 2012, pp. 1013-1019. http://dx.doi.org/10.1093/jjco/hys129

[55] J. S. Spratt, R. A. Adcock, M. Muskovin, et al., "Clinical Delivery System for Intraperitoneal Hyperthermic Chemotherapy," Cancer Research, Vol. 40, No. 2, 1980, pp. 256-260.

[56] E. P. Armour, D. McEachern, Z. Wang, et al., "Sensitivity of Human Cells to Mild Hyperthermia," Cancer Research, Vol. 53, No. 12, 1993, pp. 2740-2744.

[57] B. A. Teicher, C. D. Kowal, K. A. Kennedy, et al., "Enhancement by Hyperthermia of the in Vitro Cytotoxicity of Mitomycin C toward Hypoxic Tumor Cells," Cancer Research, Vol. 41, No. 3, 1981, pp. 1096-1099.

[58] X. J. Yang, C. Q. Huang, T. Suo, et al., "Cytoreductive Surgery Plus Hyperthermic Intraperitoneal Chemotherapy Improves Survival of Patients with Peritoneal Carcinomatosis from Gastric Cancer: Final Results of a Phase III Randomized Clinical Trial," Annals of Surgical Oncology, Vol. 18, No. 6, 2011, pp. 1575-1581. http://dx.doi.org/10.1245/s10434-011-1631-5

[59] H. R. Rosen, G. Jatzko, S. Repse, et al., "Adjuvant Intraperitoneal Chemotherapy with Carbon-Absorbed Mitomycin in Patients with Gastric Cancer: Results of Randomized Multicenter Trial of the Austrian Working Group for Surgical Oncology," Journal of Clinical Oncology, Vol. 16, No. 8, 1998, pp. 2733-2738.

[60] C. Kunisaki, H. Shimada, M. Nomura, et al., "Lack of Efficacy of Prophylactic Continuous Hyperthermic Peritoneal Perfusion on Subsequent Peritoneal Recurrence and Survival in Patients with Advanced Gastric Cancer," 
Surgery, Vol. 131, No. 5, 2002, pp. 521-528. http://dx.doi.org/10.1067/msy.2002.123769

[61] Y. Kodera, H. Nakanishi, Y. Yamamura, et al., "Prognostic Value and Clinical Implications of Disseminated Carcinoma Cells in the Peritoneal Cavity Detected by Reverse Transcriptase-Polymerase Chain Reaction and Cytology," International Journal of Cancer, Vol. 79, No. 4, 1998, pp. 429-433.

http://dx.doi.org/10.1002/(SICI)1097-0215(19980821)79: 4<429::AID-IJC20>3.0.CO;2-Z

[62] S. Shimada, E. Tanaka, T. Marutsuka, et al., "Extensive Intraoperative Peritoneal Lavage and Chemotherapy for Gastric Cancer Patients with Peritoneal Free Cancer Cells," Gastric Cancer, Vol. 5, No. 3, 2002, pp. 168-172. http://dx.doi.org/10.1007/s101200200029

[63] M. Kuramoto, S. Shimada, S. Ikeshima, et al., "Extensive Intraoperative Peritoneal Lavage as a Standard Prophylactic Strategy for Peritoneal Recurrence in Patients with Gastric Carcinoma," Annals of Surgery, Vol. 250, No. 2, 2009, pp. 242-246.

http://dx.doi.org/10.1097/SLA.0b013e3181b0c80e

[64] Y. Kodera, S. Ito, Y. Mochizuki, et al., "Long-Term Follow Up of Patients Who Were Positive for Peritoneal Lavage Cytology: Final Report from the CCOG0301 Study," Gastric Cancer, Vol. 15, No. 3, 2012, pp. 335337. http://dx.doi.org/10.1007/s10120-012-0156-3

[65] P. Jacquet and P. H. Sugarbaker, "Clinical Research Methodologies in Diagnosis and Staging of Patients with Peritoneal Carcinomatosis," In: P. H. Sugarbaker, Ed., Peritoneal Carcinomatosis: Principles of Management, Kluwer Academic Publishers, Boston, 1996, pp. 359-374. http://dx.doi.org/10.1007/978-1-4613-1247-5 23

[66] Y. Yonemura, A. Elnemr, Y. Endou, et al. "Multidisciplinary Therapy for Treatment of Patients with Peritoneal Carcinomatosis from Gastric Cancer," World Journal of Gastrointestinal Oncology, Vol. 2, No. 2, 2010, pp. 8597. http://dx.doi.org/10.4251/wjgo.v2.i2.85

[67] P. H. Sugarbaker, "Peritonectomy Procedures," Annals of Surgery, Vol. 221, No. 1, 1995, pp. 29-42. http://dx.doi.org/10.1097/00000658-199501000-00004

[68] P. H. Sugarbaker and K. A. Jablonski, "Prognostic Feature of 51 Colorectal and 130 Appendiceal Cancer Patients with Peritoneal Carcinomatosis Treated by Cytoreductive Surgery and Intraperitoneal Chemotherapy," Annals of Surgery, Vol. 221, No. 2, 1995, pp. 124-132. http://dx.doi.org/10.1097/00000658-199502000-00002

[69] A. J. Witkamp, E. de Bree, M. M. Kaag, et al., "Extensive Surgical Cytoreduction and Intraoperative Hyperthermic Intraperitoneal Chemotherapy in Patients with Pseudomyxoma Peritonei," British Journal of Surgery, Vol. 88, No. 3, 2001, pp. 458-463. http://dx.doi.org/10.1046/j.1365-2168.2001.01701.x

[70] T. Fujimura, Y. Yonemura, H. Nakagawara, et al., "Subtotal Peritonectomy with Chemohyperthermic Peritoneal Perfusion for Peritonitis Carcinomatosa in Gastrointestinal Cancer," Oncology Reports, Vol. 7, No. 4, 2000, pp. 809-814.

[71] M. Markman, "Intraperitoneal Chemotherapy," Seminars in Oncology, Vol. 18, No. 3, 1991, pp. 248-254.

[72] H. Tsujimoto, A. Hagiwara, M. Shimotsuma, et al., "Role of Milky Spots as Selective Implantation Site for Malignant Cell in Peritoneal Dissemination in Mice," Journal of Cancer Research and Clinical Oncology, Vol. 122, No. 10, 1996, pp. 590-595. http://dx.doi.org/10.1007/BF01221190

[73] H. Ishigami, J. Kitayama, S. Kaisaki, et al., "Phase II Study of Weekly Intravenous and Intraperitoneal Paclitaxel Combined with S-1 for Advanced Gastric Cancer with Peritoneal Metastasis," Annals of Oncology, Vol. 21, No. 1, 2010, pp. 67-70. http://dx.doi.org/10.1093/annonc/mdp260

[74] S. Fushida, J. Kinoshita, M. Kaji, et al., "Phase I/II Study of Intraperitoneal Docetaxel Plus S-1 for the Gastric Cancer Patients with Peritoneal Carcinomatosis," Cancer Chemotherapy and Pharmacology, Vol. 71, No. 5, 2013, pp. 1265-1272. http://dx.doi.org/10.1007/s00280-013-2122-0 